\title{
On the Semi-Classical Vacuum Structure of the Electroweak Interaction
}

\author{
Jürgen Tolksdorf* \\ Inst. of Mathematics \\ University of Mannheim, Germany
}

Oct. 17, 2003

\begin{abstract}
It is shown that in the semi-classical approximation of the electroweak sector of the Standard Model the moduli space of vacua can be identified with the first de Rham cohomology group of space-time. This gives a slightly different physical interpretation of the occurrence of the well-known Ahoronov-Bohm effect. Moreover, when charge conjugation is taken into account, the existence of a non-trivial ground state of the Higgs boson is shown to be equivalent to the triviality of the electroweak gauge bundle. As a consequence, the gauge bundle of the electromagnetic interaction must also be trivial. Though derived at "tree level" the results presented here may also have some consequences for quantizing, e. g., electromagnetism on an arbitrary curved space-time.
\end{abstract}

*email: tolkdorf@euler.math.uni-mannheim.de 


\section{Introduction}

We consider as a specific Yang-Mills-Higgs (YMH) gauge theory the bosonic part of the electroweak interaction of the Standard Model of particle physics. Its basic geometrical objects are given by a $\mathrm{SU}(2) \times \mathrm{U}(1)$ gauge potential $A=W+B \in \Omega^{1}\left(\mathcal{M}, \mathbb{R}^{3} \oplus \mathbb{R}\right)$ together with a complex vector field $\Phi \in \Omega^{0}\left(\mathcal{M}, \mathbb{C}^{2}\right)$. Here, $\mathcal{M}$ denotes a space-time manifold which is usually identified with Minkowski space $\mathbb{R}^{1,3}$. Like in perturbation theory, the physical interpretation of the pair $(A, \Phi)$ is that of a "fluctuation of the (classical) bosonic vacuum" $(A=0, \Phi=0)$ via the known replacement

$$
\begin{aligned}
d & \mapsto d_{\mathrm{A}}:=d+A, \\
\mathbf{z}_{0} & \mapsto \phi:=\mathbf{z}_{0}+\Phi,
\end{aligned}
$$

where $\mathbf{z}_{0} \in \mathbb{C}^{2}$ is a chosen minimum of the Higgs potential $V_{\mathrm{H}}:=\lambda|\mathbf{z}|^{4}-\mu^{2}|\mathbf{z}|^{2}(\lambda, \mu>0)$ and " $d$ " is the exterior derivative.

From a geometrical point of view, a (classical) bosonic vacuum of the electroweak interaction may be represented by the canonical YMH pair

$$
\left(\Theta_{0}, \mathcal{V}_{0}\right)
$$

where $\mathcal{V}_{0}$ is the canonical mapping

$$
\begin{aligned}
\mathcal{V}_{0}: \mathcal{M} & \longrightarrow \mathcal{M} \times \operatorname{orbit}\left(\mathbf{z}_{0}\right) \\
x & \mapsto\left(x, \mathbf{z}_{0}\right)
\end{aligned}
$$

and $\Theta_{0}$ is the flat connection on $\operatorname{pr}_{1}: \mathcal{M} \times \mathbb{C}^{2} \rightarrow \mathcal{M}$ associated with the canonical connection $\operatorname{pr}_{2}^{*} \zeta^{\mathrm{MC}}$ on the trivial principal $\mathrm{SU}(2) \times \mathrm{U}(1)$ bundle

$$
\begin{aligned}
\operatorname{pr}_{1}: \mathcal{M} \times(\mathrm{SU}(2) \times \mathrm{U}(1)) & \longrightarrow \mathcal{M} \\
p=\left(x, g \equiv\left(g_{(2)}, g_{(1)}\right)\right) & \mapsto x .
\end{aligned}
$$

Here respectively, $\zeta^{\mathrm{MC}}$ is the Maurer-Cartan form on $\mathrm{SU}(2) \times \mathrm{U}(1), \operatorname{pr}_{2}(x, g):=g$ and $\operatorname{orbit}\left(\mathbf{z}_{0}\right) \subset \mathbb{C}^{2}$ denotes the orbit of $\mathbf{z}_{0}$ with respect to the unitary representation $\rho_{\mathrm{H}}(g):=g_{(2)} g_{(1)}^{\mathrm{y}}(\mathrm{y} \in \mathbb{Q})$. Notice that the specific Higgs potential $\mathrm{V}_{\mathrm{H}}$ in the Standard Model has only one such orbit that is homeomorphic to $\mathrm{S}^{3} \subset \mathbb{R}^{4}$.

The canonical YMH pair (2) represents a specific absolute minimum of the energy functional associated with the known $\mathrm{YMH}$ action $\mathcal{I}_{\mathrm{YMH}}=\mathcal{I}_{\mathrm{YM}}+\mathcal{I}_{\mathrm{H}}$. Of course, any 
gauge equivalent YMH pair contains the same physical information. A question that naturally follows is how many gauge inequivalent vacua of the electroweak interaction exist and what is their physical meaning? Another question closely tied to the previous one is: How do we know that the gauge bundle underlying the electroweak interaction is actually trivial? In other words, what can we learn from the study of the moduli space of vacua concerning the topology of space-time and the gauge bundle? At a first glance this question may sound like being of purely mathematical interest. However, the existence of gauge inequivalent ground states, and tied to it the topology of space-time and of the gauge bundle, may also have consequences with respect to the quantization of a spontaneously broken gauge theory. For instance, when trying to quantize electromagnetism on an arbitrary (globally hyperbolic) space-time manifold $\mathcal{M}$ one has to consider the non-triviality of $\mathrm{H}_{\mathrm{deR}}^{2}(\mathcal{M})$. However, when seen from a gauge geometrical viewpoint, the Maxwell-Faraday equation $d F_{\text {elm }}=0$ becomes an identity (the "Bianchi Identity"). That is, the electromagnetic field strength $F_{\text {elm }} \in \Omega^{2}(\mathcal{M})$ is considered as the local pull-back of the curvature of a connection form $\omega \in \Omega^{1}(\mathrm{Q})$ on the underlying electromagnetic gauge bundle $\mathcal{Q}: \pi_{\mathrm{Q}}: \mathrm{Q} \rightarrow \mathcal{M}$. In other words, $F_{\text {elm }}=d \sigma_{\alpha}^{*} \omega$, where $\sigma_{\alpha}: \mathcal{M} \supset \mathrm{U}_{\alpha} \rightarrow \mathrm{Q}$ is a local trivialization of $\mathcal{Q}$. Therefore, in order to quantize the electromagnetic gauge potentials $A_{\alpha} \equiv \sigma_{\alpha}^{*} \omega$ one not only has to take into account the topology of space-time $\mathcal{M}$ but, in particular, the topology of the electromagnetic gauge bundle $\mathcal{Q}$. In fact, if the latter turns out to be trivial, then every gauge potential $A \in \Omega^{1}(\mathcal{M})$ is a globally defined object independent of the topology of space-time.

Thus, also from a physical perspective it seems appropriate to put the above geometrical interpretation of a vacuum (or its "fluctuation") in a more general geometrical perspective. A corresponding discussion of a general (classical) bosonic vacuum can be found in [Tolk'03(a)] (for a discussion of the fermionic vacuum, please see [Tolk'03(b)]). There, we also discussed the geometrical meaning of the bosonic (resp. the fermionic) "mass matrix" and the existence of the "unitary gauge". For the convenience of the reader we shall summarize in the next section the basic geometrical notions used afterwards to prove that in the case of the electroweak interaction the moduli space of bosonic vacua is nonempty iff the electromagnetic gauge bundle is trivial. Moreover, in this case the principal $\mathrm{SU}(2) \times \mathrm{U}(1)$ bundle underlying the electroweak interaction is also trivial and the moduli space of vacua consists of only one point, which is represented by $\left(\Theta_{0}, \mathcal{V}_{0}\right)$ iff the first de Rham cohomology group of space-time is trivial. Thus, 
if electromagnetism is supposed to be dynamically generated by spontaneous symmetry breaking, the corresponding principal U(1) bundle representing the electromagnetic interaction must be trivial. Besides the usual assumptions of being paracompact, Hausdorff, orientable and smooth this statement turns out to be independent of the topology of space-time and independent of its geometry.

Terminology: In the following we would like to briefly comment on why it might be useful to use a global geometrical description of what is usually referred to as an "elementary particle". Also, these remarks serve to clarify the physical terminology used in this paper.

In classical physics "particles" are geometrically represented by timelike (future oriented) one-dimensional submanifolds of a given space-time $\mathcal{M}$. In contrast, in the semi-classical approximation of a (quantum) field theoretical description of a "particle" the latter is usually identified by its state, described by a (quantum) field. Such an identification seems inappropriate since, for example, the same particle may approach different states. In particular, within the realm of gauge theories the state of a particle is supposed to be a gauge dependent concept and thus is of no direct physical meaning. Moreover, in a quantum field theoretical description of a "particle" the notion of the latter becomes even more subtle for particles may be "created", "annihilated" or "transformed into each other". Consequently, physical notions like "mass" or "charge" usually refer to "asymptotically free particles". However, how one can make the latter geometrically precise within the context of gauge theories for "freeness" means no interaction and thus seems to be a gauge dependent concept? Also, in particle physics certain asymptotically free particles are considered to perform a "particle multiplet" which transforms according to some (unitary) representation of a given "gauge group" G. Again, when seen from a gauge geometrical viewpoint such an interpretation of the "internal space" always refers to a (local) trivialization of the gauge bundle with structure group G. However, since a (local) trivialization of a gauge bundle $\mathcal{P}$ cannot be performed experimentally such a description of asymptotically free particles within gauge theories seems spurious. Notice that this is quite in contrast to relativity, where the mathematical concept of a local trivialization has a direct physical meaning and so the typical fiber of the tangent bundle of space-time, too. As a consequence, the concept of an asymptotically free particle should be a purely geometrical one. It seems natural to geometrically describe elementary particles, at least in a "semi-classical 
approximation" of a quantum theory, as (isomorphism classes of) Hermitian vector bundles $\xi$ (see, for instance, [Derd'92]). The possible states of a particle may then be represented by sections of the appropriate bundles. In contrast to a "particle" the state of the latter can still be considered as a local concept. The gauge interaction between various particles is modeled by the assumption that the vector bundles are associated with a given principal G-bundle $\mathcal{P}$.

For instance, in the case of the bosonic sector of the electroweak interaction the "particle content" of the latter is known not to be given by $(W, B, \Phi)$ but instead by the "electromagnetic gauge boson" $A_{\text {elm }}$ together with the massive and electrically (un-)charged "weak vector bosons" $Z^{0}, W^{ \pm}$and the "physical Higgs boson" $\Phi_{\mathrm{H}, \mathrm{phys}}$. Here,

$$
\begin{aligned}
A_{\text {elm }} & :=\cos \theta_{\mathrm{w}} B+\sin \theta_{\mathrm{w}} W_{3}, \\
Z^{0} & :=\cos \theta_{\mathrm{w}} W_{3}-\sin \theta_{\mathrm{w}} B, \\
W^{ \pm} & :=W_{1} \pm i W_{2}
\end{aligned}
$$

with $W=\left(W_{1}, W_{2}, W_{3}\right) \in \Omega^{1}\left(\mathcal{M}, \mathbb{R}^{3}\right)$ the "weak gauge boson" and $\Phi_{\mathrm{H}, \mathrm{phys}}$ the "physical component" of the "Higgs boson" $\Phi=\left(\Phi_{\mathrm{G}}, \Phi_{\mathrm{H}, \mathrm{phys}}\right) \in \Omega^{0}\left(\mathcal{M}, \mathbb{R}^{4}\right)$. In the semi-classical approximation a common usage of terminology in particle physics is that physically non-interacting particles are identified with "free fields" on space-time $\mathcal{M}$ (see standard texts, for example, [Aitch/Hey'82], [Nach'89], or Chapter 21.3 in [Wein'01]). However, neither the definition (5), nor the notion of a "free field", in general cannot be gauge invariantly defined. One may thus ask for the geometrical meaning of the "particle content"

$$
\left(A_{\text {elm }}, Z^{0}, W^{ \pm}, \Phi_{\mathrm{H}, \text { phys }}\right)
$$

of the (bosonic part of the) electroweak sector of the Standard Model.

As it turns out, the "free particles" (6) are intimately related to the notion of a bosonic vacuum of the electroweak interaction. Also, the "free particles" actually have a simple geometrical meaning. Indeed, we shall show how the particle content (6) can geometrically be considered as real line bundles over space-time which naturally come with the geometry of spontaneous symmetry breaking of the electroweak interaction. Moreover, these line bundles define the extrinsic curvature of the vacuum geometrically considered as specific submanifolds. As one may expect, these extrinsic curvatures are 
proportional to the masses of the bosons.

However, given such a global description of an elementary particle one may ask about the topological structure of the bundles $\xi$. Since they are associated bundles, this raises the question about the topology of the underlying gauge bundle $\mathcal{P}$ which, of course, is closely linked to the topology of space-time $\mathcal{M}$ itself. In elementary particle physics one usually encounters $\mathcal{M} \simeq \mathbb{R}^{1,3}$. Of course, this specific assumption leads to a definite answer concerning the topology of $\mathcal{P}$ and thus of $\xi$. However, as we have mentioned before when trying to quantize electromagnetism on a general space-time $\mathcal{M}$, one has to consider $d F_{\text {elm }}=0$ which, in general, gives rise only to the local existence of an electromagnetic gauge potential $A_{\text {elm }}$. On the other hand, if $\mathcal{P}$ is supposed to be trivial, then every gauge potential can be considered as a globally defined object. But what do we know about the topology of the underlying gauge bundle? Since the latter has no direct physical meaning it seems inappropriate to make any a priori assumptions with respect to the topology of $\mathcal{P}$. Therefore, our "strategy" is the following; the topology of $\mathcal{P}$ is supposed to be arbitrary but fixed, analogous to the assumption of an arbitrary but fixed space-time background $\mathcal{M}$. Then, we try to use physically well-established assumptions in order to restrict the topological structure of both space-time and of the gauge bundle. In the present paper the physically well-motivated assumptions made, for example, basically consist in the existence of a non-trivial ground state of the Higgs boson and in the assumption that the $\mathrm{W}^{ \pm}$-vector bosons of the weak interaction are charge conjugate to each other. As we shall see these two physical assumptions fully fix the topology of the bundles under consideration.

\section{The geometrical setup}

In this section we summarize the basic geometrical notions which are used to generalize $\left(\Theta_{0}, \mathcal{V}_{0}\right)$ to the case of arbitrary principal G-bundles $\mathcal{P}$

$$
\begin{aligned}
\pi_{\mathrm{P}}: \mathrm{P} & \longrightarrow \mathcal{M} \\
p & \mapsto x
\end{aligned}
$$

Here, $\mathrm{G}$ denotes a finite dimensional compact, semi-simple real Lie group and $\left(\mathcal{M}, g_{\mathrm{M}}\right)$ a smooth semi-Riemannian manifold of arbitrary signature. Topologically, $\mathcal{M}$ is supposed to be paracompact, Hausdorff and orientable. Notice that, like the (semi-)Riemannian structure $g_{\mathrm{M}}$, the bundle structure of $\mathcal{P}$ is supposed to be given but otherwise 
arbitrary.

Then, a YMH-gauge theory can be characterized by the following data

$$
\left(\mathcal{P}, \rho_{\mathrm{H}}, \mathrm{V}_{\mathrm{H}}\right),
$$

where $\rho_{\mathrm{H}}: \mathrm{G} \rightarrow \mathrm{GL}(\mathrm{N}, \mathbb{C})$ is a unitary representation and $\mathrm{V}_{\mathrm{H}}: \mathbb{C}^{\mathrm{N}} \rightarrow \mathbb{R}$ denotes a G-invariant smooth function that is bounded from below. Moreover, its Hessian is supposed to be positive definite transversally to the orbit of each minimum of $V_{H}$. Accordingly, we call such a function $\mathrm{V}_{\mathrm{H}}$ a "generalized Higgs potential".

Naturally associated with the data (8) are two Hermitian vector bundles; the "Higgs bundle" $\xi_{\mathrm{H}}$ and the "Yang-Mills bundle" $\xi_{\mathrm{YM}} \equiv \tau_{\mathrm{M}}^{*} \otimes \mathfrak{a} \mathfrak{d}(\mathcal{P})$. Here, the Higgs bundle is defined by

$$
\begin{aligned}
\pi_{\mathrm{H}}: \mathrm{E}_{\mathrm{H}}:=\mathrm{P} \times_{\rho_{\mathrm{H}}} \mathbb{C}^{\mathrm{N}} & \longrightarrow \mathcal{M} \\
\mathfrak{z} \equiv[(p, \mathbf{z})] & \mapsto \pi_{\mathrm{P}}(p)
\end{aligned}
$$

and the YM bundle as the tensor product of the cotangent bundle $\tau_{\mathrm{M}}^{*}$ of $\mathcal{M}$ with the "adjoint bundle" ad $(\mathcal{P})$

$$
\begin{aligned}
\pi_{\mathrm{ad}}: \operatorname{ad}(\mathrm{P}):=\mathrm{P} \times_{\mathrm{ad}} \operatorname{Lie}(\mathrm{G}) & \longrightarrow \mathcal{M} \\
\tau \equiv[(p, \mathrm{~T})] & \mapsto \pi_{\mathrm{P}}(p) .
\end{aligned}
$$

The Higgs bundle and the Yang-Mills bundle are regarded to geometrically represent, respectively, the Higgs boson and the Yang-Mills boson. Accordingly, one may physically interpret the sections of these bundles as the states of the respective bosons.

Each minimum $\mathbf{z}_{0} \in \mathbb{C}^{\mathrm{N}}$ of the Higgs potential gives rise to a specific fiber subbundle $\xi_{\text {orb }}$ of the Higgs bundle called the "Orbit bundle" with respect to the minimum $\mathbf{z}_{0}$. It is defined by

$$
\begin{aligned}
\pi_{\text {orb }}: \mathcal{O} \operatorname{rbit}\left(\mathbf{z}_{0}\right):=\mathrm{P} \times_{\rho_{\text {orb }}} \operatorname{orbit}\left(\mathbf{z}_{0}\right) & \longrightarrow \mathcal{M} \\
\mathfrak{z} \equiv[(p, \mathbf{z})] & \mapsto \pi_{\mathrm{P}}(p),
\end{aligned}
$$

where $\rho_{\text {orb }}:=\left.\rho_{\mathrm{H}}\right|_{\text {orbit }\left(\mathbf{z}_{0}\right)}$. Notice that $\xi_{\text {orb }} \simeq \xi_{\text {orb }}^{\prime}$ iff $\mathbf{z}_{0}^{\prime}$ and $\mathbf{z}_{0}$ are on the same orbit. Like the gauge bundle $\mathcal{P}$, the orbit bundle has no direct physical meaning. However, since $\xi_{\text {orb }} \subset \xi_{\mathrm{H}}$, any section $\mathcal{V}$ of the orbit bundle physically represents a possible ground state of the Higgs boson. We therefore call $\mathcal{V}$ a "vacuum section". We denote by $\mathcal{H} \subset \mathcal{G}$ the 
invariance group of the vacuum section. It is a closed subgroup of the gauge group $\mathcal{G}$ of $\mathcal{P}$ and may point-wise be identified with the isotropy group $\mathrm{I}\left(\mathbf{z}_{0}\right) \subset \mathrm{G}$ of the minimum $\mathbf{z}_{0}$.

Every vacuum section singles out a specific class of connections on $\mathcal{P}$. For this we remark that each vacuum section $\mathcal{V}$ is in one-to-one correspondence to an "H-reduction" $(\mathcal{Q}, \iota)$ of the principal G-bundle $\mathcal{P}$ (see, e. g., [Koba/Nomi'96]). That is, there is a unique principal H-bundle $\mathcal{Q}$

$$
\begin{aligned}
\pi_{\mathrm{Q}}: \mathrm{Q} & \longrightarrow \mathcal{M} \\
q & \mapsto x
\end{aligned}
$$

together with a bundle embedding $\iota: \mathcal{Q} \hookrightarrow \mathcal{P}\left(\right.$ i. e. $\pi_{\mathrm{P}}(\iota(q))=\pi_{\mathrm{Q}}(q)$ for all $\left.q \in \mathrm{Q}\right)$, such that $\mathrm{H} \simeq \mathrm{I}\left(\mathbf{z}_{0}\right)$. Indeed, in contrast to the more physically intuitive notion of a vacuum section the usually geometrical description of spontaneous symmetry breaking only refers to the notion of a bundle reduction (see, e. g., [Blee'81], [Choq/deWit'89], [Ster'95], [Trau'80].)

Note that, in general, the principal H-bundle $\mathcal{Q}$ will be non-trivial even if the principal G-bundle $\mathcal{P}$ is equivalent to the trivial one. Of course, the triviality of $\mathcal{Q}$ implies the triviality of $\mathcal{P}$. Also, any connection on $\mathcal{Q}$ generally induces a connection on $\mathcal{P}$ but not vice versa. A connection $\mathcal{A}$ on $\mathcal{P}$ is said to be "H-reducible" iff $\iota^{*} \mathcal{A}$ is also a connection on $\mathcal{Q}$. In this case we call $\mathcal{A}$ compatible with the appropriate vacuum section $\mathcal{V}$. A simple criterion for a connection to be compatible with a vacuum section is given by the following

Lemma 2.1 A connection on $\mathcal{P}$ is compatible with a vacuum section $\mathcal{V} \in \Gamma\left(\xi_{\text {orb }}\right)$ iff the associated connection $\mathcal{A} \in \mathcal{A}\left(\xi_{\mathrm{H}}\right)$ on the Higgs bundle satisfies

$$
d_{\mathrm{A}} \mathcal{V}=0
$$

with $d_{\mathrm{A}}$ the exterior covariant derivative with respect to $\mathcal{A}$.

Proof: Since a connection on $\mathcal{P}$ is H-reducible iff the corresponding connection form on $\mathcal{P}$ takes values in $\operatorname{Lie}(\mathrm{H})$ the statement follows from $\mathcal{V}(x)=\left.\left[\left(\iota(q), \mathbf{z}_{0}\right)\right]\right|_{q \in \pi_{\mathrm{Q}}^{-1}(x)}$. 
We call a YMH pair $(\Theta, \mathcal{V}) \in \mathcal{A}\left(\xi_{\mathrm{H}}\right) \times \Gamma\left(\xi_{\mathrm{H}}\right)$ a (classical) "bosonic vacuum" (or, in the context of this paper, an "electroweak vacuum") iff $\mathcal{V}$ denotes a vacuum section with respect to some chosen minimum $\mathbf{z}_{0}$ and $\Theta$ a connection on $\xi_{\mathrm{H}}$ associated with a flat connection on $\mathcal{P}$ and which is compatible with $\mathcal{V}$.

The notion of a (classical) bosonic vacuum introduced here indeed generalizes the geometrical interpretation of a vacuum as described in the introduction. Since in the case of $\left(\mathcal{M}, g_{\mathrm{M}}\right) \simeq \mathbb{R}^{1,3}$ it follows that both $\mathcal{P}$ and $\mathcal{Q}$ must be trivial for any vacuum section. The latter may then be identified with smooth mappings $\nu: \mathcal{M} \rightarrow \operatorname{orbit}\left(\mathbf{z}_{0}\right)$. Moreover, any such mapping is easily shown to be gauge equivalent to the canonical mapping $\mathcal{V}_{0}$ which corresponds to the canonical embedding

$$
\begin{aligned}
\mathcal{M} \times \mathrm{H} & \hookrightarrow \mathcal{M} \times \mathrm{G} \\
(x, h) & \mapsto(x, h) .
\end{aligned}
$$

In [Tolk'03(a)] it is shown that one encounters a similar situation in the case where $\pi_{1}(\mathcal{M})=0$. More precisely, in the given reference it is proved that on a simply connected manifold $\mathcal{M}$ there exists at most one vacuum for each orbit. Moreover, these vacua are all gauge equivalent to $\left(\Theta_{0}, \mathcal{V}_{0}\right)$. It is then a natural question to ask for the structure of the moduli space of vacua in the case of $\pi_{1}(\mathcal{M}) \neq 0$. This will be done in the next section for the particular case of the electroweak interaction.

We close this section with the remark that with respect to any vacuum section $\mathcal{V}$ the (realification of the) Higgs bundle decomposes into the Whitney sum of two real subbundles called the "Goldstone bundle" $\xi_{\mathrm{G}}$ and the "physical Higgs bundle" $\xi_{\mathrm{H}, \mathrm{phys}}$, i. e.

$$
\xi_{\mathrm{H}}=\xi_{\mathrm{G}} \oplus \xi_{\mathrm{H}, \mathrm{phys}} .
$$

Moreover, since a vacuum section can be considered as a specific embedding of space-time into the total space of the Higgs bundle, the tangent bundle of $\mathcal{M}$ together with the Goldstone and the physical Higgs bundle build a "global 3-Bein" along the vacuum $\mathcal{V}(\mathcal{M}) \subset \mathrm{E}_{\mathrm{H}}$. In particular, the physical Higgs bundle $\xi_{\mathrm{H}, \mathrm{phys}}$ can be identified with the normal bundle of $\mathcal{O} \operatorname{rbit}\left(\mathbf{z}_{0}\right) \subset \mathrm{E}_{\mathrm{H}}$ restricted to $\mathcal{V}(\mathcal{M}) \subset \mathrm{E}_{\mathrm{H}}$. Notice that the 3 -Bein is orthogonal with respect to the metric $g_{\mathrm{H}}$ induced by $\left(\Theta, g_{\mathrm{M}}\right)$ on $\mathrm{E}_{\mathrm{H}}$. The exterior curvature of $\mathcal{O}$ rbit $\left(\mathbf{z}_{0}\right)$ along the vacuum is proportional to the mass of the physical Higgs boson. 


\section{The moduli space of vacua of the electroweak in- teraction}

The structure of the moduli space of the bosonic vacua is found to be surprisingly simple in the case of the electroweak interaction. This is so because this interaction turns out to have some special topological features which we will discuss in this section.

First, we again summarize the data defining the electroweak interaction as a specific YMH gauge theory. In this case $\left(\mathcal{P}, \rho_{\mathrm{H}}, \mathrm{V}_{\mathrm{H}}\right)$ is given by

- a principal $\mathrm{G}:=\mathrm{SU}(2) \times \mathrm{U}(1)$-bundle $\mathcal{P}$,

- the unitary representation $\rho_{\mathrm{H}}: \mathrm{G} \rightarrow \mathrm{GL}(2, \mathbb{C}), g \equiv\left(g_{(2)}, g_{(1)}\right) \mapsto g_{(2)} g_{(1)}^{\mathrm{y}}$ (with "hypercharge" y $\in \mathbb{Q}$ ),

- the Higgs potential $V_{\mathrm{H}}(\mathbf{z}):=\lambda|\mathbf{z}|^{4}-\mu^{2}|\mathbf{z}|^{2}(\lambda, \mu>\mathbf{0})$.

As already mentioned in the introduction, this Higgs potential has but one orbit of minima that is isomorphic to $S^{3} \subset \mathbb{C}^{2}$. Moreover, it has the special feature of being "rotationally symmetric". That is, the Higgs potential can be written as $\mathrm{V}_{\mathrm{H}}=f_{\mathrm{H}} \circ r$, with $r(\mathbf{z}):=|\mathbf{z}|$ the radial function and $f_{\mathrm{H}} \in \mathcal{C}^{\infty}\left(\mathbb{R}_{+}\right)$bounded from below. As a consequence, it can be shown that there exists a vacuum section $\mathcal{V}$ iff the Higgs bundle $\xi_{\mathrm{H}}$ admits a non-vanishing section. Moreover, with respect to such a vacuum section the physical Higgs bundle is a trivial real line bundle. Also, for any non-vanishing section $\Phi$ of the Higgs bundle one may always find a vacuum, such that $\Phi$ can be identified with a section of the corresponding physical Higgs bundle (see again [Tolk'03(a)]). In other words, there always exists a vacuum such that $\Phi$ is in the "unitary gauge" with respect to this vacuum.

Next, we prove that in the case of the electroweak interaction the adjoint bundle $\mathfrak{a} \mathfrak{d}(\mathcal{P})$ decomposes into the Whitney sum of two real line bundles and one real vector bundle of rank two.

Proposition 3.1 Let $\left(\mathcal{P}, \rho_{\mathrm{H}}, V_{\mathrm{H}}\right)$ be the data defining the electroweak interaction as a $Y M H$ gauge theory. Also, let $\mathcal{V} \in \Gamma\left(\xi_{\text {orb }}\right)$ be a vacuum section with respect to some minimum $\mathbf{z}_{0}$ of the Higgs potential. With respect to the vacuum section $\mathcal{V}$ the adjoint 
bundle $\mathfrak{a d}(\mathcal{P})$, considered as a vector bundle, decomposes as

$$
\mathfrak{a} \mathfrak{d}(\mathcal{P}) \simeq \mathfrak{a} \mathfrak{d}(\mathcal{Q}) \oplus\left(\xi_{\mathrm{z}} \oplus \xi_{\mathrm{w}}\right) .
$$

Here, $\xi_{\mathrm{z}}$ and $\xi_{\mathrm{w}}$ respectively denote a real vector bundle of rank one and of rank two.

Proof: When considered as a vector bundle $\mathfrak{a}(\mathcal{P})$ is H-reducible and decomposes as (see, [Tolk'03(a)])

$$
\mathfrak{a} \mathfrak{d}(\mathcal{P}) \simeq \mathfrak{a d}(\mathcal{Q}) \oplus \xi_{\mathrm{G}}
$$

Likewise, the Yang-Mills mass matrix

$$
\begin{aligned}
\mathcal{V}^{*} \mathrm{M}_{\mathrm{YM}}^{2}: \mathfrak{a} \mathfrak{d}(\mathcal{P}) & \longrightarrow \mathfrak{a} \mathfrak{d}(\mathcal{P}) \\
\tau \equiv[(p, \mathrm{~T})] & \mapsto \mathcal{V}^{*} \mathrm{M}_{\mathrm{YM}}^{2}(\tau),
\end{aligned}
$$

with $\mathcal{V}^{*} \mathrm{M}_{\mathrm{YM}}^{2}(\tau)(x):=\left.\left[\left(p, \operatorname{ad}_{g^{-1}}\left(\mathbf{M}_{\mathrm{YM}}^{2}\left(\mathbf{z}_{0}\right) \operatorname{ad}_{g}(\mathrm{~T})\right)\right)\right]\right|_{p \in \pi_{\mathrm{P}}^{-1}(x)}$, decomposes as

$$
\mathcal{V}^{*} \mathrm{M}_{\mathrm{YM}}^{2}=(0) \oplus \mathcal{V}^{*} \mathrm{M}_{\mathrm{YMG}}^{2},
$$

where $\mathcal{V}^{*} \mathrm{M}_{\mathrm{YMG}}^{2}:=\left.\mathcal{V}^{*} \mathrm{M}_{\mathrm{YM}}^{2}\right|_{\xi_{\mathrm{G}}}$ has maximal rank. In (18) $p=\iota(q) g$ for arbitrary $q \in \pi_{\mathrm{Q}}^{-1}(x), g \in \mathrm{G}$ and $\mathbf{M}_{\mathrm{YM}}^{2}\left(\mathbf{z}_{0}\right) \in \operatorname{End}(\operatorname{Lie}(\mathrm{G}))$ is defined by $\beta\left(\mathbf{M}_{\mathrm{YM}}^{2}\left(\mathbf{z}_{0}\right)(\mathrm{T}), \mathrm{T}^{\prime}\right)=$ $2 \rho_{\mathrm{H}}^{\prime}(\mathrm{T}) \mathbf{z}_{0} \cdot \rho_{\mathrm{H}}^{\prime}\left(\mathrm{T}^{\prime}\right) \mathbf{z}_{0}$ for all $\mathrm{T}, \mathrm{T}^{\prime} \in \operatorname{Lie}(\mathrm{G})$. The symmetric bilinear form $\beta$ on $\operatorname{Lie}(\mathrm{G})$ is given by the most general parameterized Killing form, and $\rho_{\mathrm{H}}^{\prime}$ denotes the real form of the "derived" representation (see again, loc sit). Note that $\mathcal{V}^{*} \mathrm{M}_{\mathrm{YM}}^{2}$ has constant spectrum and lays within the commutant of the reduced gauge group $\mathcal{H}$. Moreover, the spectrum only depends on the orbit of $\mathbf{z}_{0}$ and not on the vacuum section $\mathcal{V}$ chosen. From the above follows that one can decompose the Goldstone bundle $\xi_{\mathrm{G}}$ into the eigenbundles of the Yang-Mills mass matrix. Since the latter commutes with the (representation of the) electromagnetic gauge group, the spectrum of $\mathcal{V}^{*} \mathrm{M}_{\mathrm{YM}}^{2}$ consists of maximally two different eigenvalues $\mathrm{m}_{\mathrm{z}}, \mathrm{m}_{\mathrm{W}} \in \mathbb{R}_{+}$. If we denote by $\rho_{\mathrm{G}}$ the restriction of the real form of $\rho_{\mathrm{H}}$ to the typical fiber of the Goldstone bundle, then $\rho_{\mathrm{G}}(h)=\mathbf{A} \oplus(1)$ for all $h \in \mathrm{H}$ (where $\mathbf{A} \in \mathrm{SO}(2)$ ). Therefore, the Goldstone bundle decomposes into the Whitney sum of a real rank one vector bundle $\xi_{z}$, which corresponds to the eigenvalue $\mathrm{m}_{\mathrm{z}}$, and a rank two vector bundle $\xi_{\mathrm{w}}$, which corresponds to the eigenvalue $\mathrm{m}_{\mathrm{w}}$ of the Yang-Mills mass matrix.

The $\mathcal{V}$-induced isomorphism (17) can be considered as a geometrical variant of what is called the "Higgs Dinner" (see, [Hig'64]). As a consequence, in the case of the 
electroweak interaction the Yang-Mills bundle decomposes as

$$
\xi_{\mathrm{YM}} \simeq \xi_{\mathrm{elm}} \oplus\left(\xi_{\mathrm{W}^{ \pm}} \oplus \xi_{\mathrm{z}^{0}}\right),
$$

where $\xi_{\text {elm }}:=\tau_{\mathrm{M}}^{*} \otimes \mathfrak{a d}(\mathcal{Q})$ geometrically represents the electromagnetic gauge boson and $\xi_{\mathrm{Z}^{0}}:=\tau_{\mathrm{M}}^{*} \otimes \xi_{\mathrm{z}}$ the massive electromagnetically neutral $\mathrm{Z}^{0}$ vector boson of the weak interaction. Note that $\mathfrak{a} \mathfrak{d}(\mathcal{Q})$ and $\xi_{\mathrm{z}}$ are trivial, for they are "uncharged" (i. e. they carry the trivial representation of the electromagnetic gauge group $\mathcal{H}$ ).

The rank two vector bundle

$$
\xi_{\mathrm{w}^{ \pm}}:=\tau_{\mathrm{M}}^{*} \otimes \xi_{\mathrm{w}}
$$

geometrically represents a massive electrically charged vector boson. Indeed, one may naturally identify the real vector bundle $\xi_{\mathrm{w}}$ of rank two with the complex line bundle associated with the electromagnetic gauge bundle $\mathcal{Q}$ via the fundamental representation of $\mathrm{H}$. Physically one can thus identify (21) either with the $W^{+}$- or with the $W^{-}$-boson of the weak interaction. Because $\mathbf{A} \in \mathrm{SO}(2)$ has no real eigenvalues the real vector bundle $\xi_{\mathrm{w}}$ does not naturally decompose into two real line bundles that geometrically represent the $W^{+}$- and the $W^{-}$-boson. The motivation for nonetheless identifying $\xi_{\mathrm{w}^{ \pm}}$with either of the well-known massive electrically charged vector bosons is as follows: Considered as a complex vector bundle of rank two $\mathbb{C} \otimes \xi_{\mathrm{w}}$ decomposes into

$$
\mathbb{C} \otimes \xi_{\mathrm{w}}=\xi_{\mathrm{w}^{+}} \oplus \xi_{\mathrm{w}^{-}} .
$$

Here, either $\xi_{\mathrm{w}^{+}}$is assumed to carry the fundamental representation of the electromagnetic gauge group and then $\xi_{\mathrm{w}^{-}}=\overline{\xi_{\mathrm{w}^{+}}}$or vice versa. Both complex line bundles are also eigenbundles of the Yang-Mills mass matrix with respect to the eigenvalue $m_{w}$. Since $\xi_{\mathrm{w}}$ carries the fundamental representation of the (real form of the) electromagnetic gauge group one may naturally identify $\xi_{\mathrm{w}}$ either with $\xi_{\mathrm{w}^{+}}$or with $\xi_{\mathrm{w}^{-}}$.

As mentioned before, to geometrically represent both the $W^{+}$- and the $W^{-}$-boson as subbundles of the Yang-Mills bundle one needs additional structure. Physically, this additional piece of input arises from the assumption that the $W^{ \pm}$-bosons are charge conjugate to each other. Since in the case at hand charge conjugation is the same as complex conjugation on the complex line bundle $\xi_{\mathrm{w}}$, charge conjugation geometrically means that there exists a real line bundle such that its complexification equals $\xi_{\mathrm{w}}$. In other words, to assume that the $W^{ \pm}$-bosons of the weak interaction are charge 
conjugate to each other is the same as to assume that there exist real line bundles $\xi_{\mathrm{w}_{1}} \simeq \xi_{\mathrm{w}_{2}}$, such that

$$
\xi_{\mathrm{w}}=\xi_{\mathrm{w}_{1}} \oplus \xi_{\mathrm{w}_{2}} .
$$

In this case, the Yang-Mills mass matrix $\mathcal{V}^{*} \mathrm{M}_{\mathrm{YM}}^{2}$ together with the real structure $\mathcal{J}$ (complex conjugation) on $\xi_{\mathrm{w}}$ permits to decompose the Yang-Mills bundle into the Whitney sum of four real line bundles

$$
\xi_{\mathrm{YM}}=\xi_{\mathrm{elm}} \oplus\left(\xi_{\mathrm{Z}^{0}} \oplus \xi_{\mathrm{W}_{1}} \oplus \xi_{\mathrm{W}_{2}}\right),
$$

where

$$
\begin{aligned}
& \xi_{\mathrm{w}^{+}}=\xi_{\mathrm{w}_{1}} \oplus i \xi_{\mathrm{w}_{2}}, \\
& \xi_{\mathrm{w}^{-}}=\xi_{\mathrm{w}_{1}} \ominus i \xi_{\mathrm{w}_{2}} .
\end{aligned}
$$

Note that it is a well-established empirical fact that the electromagnetic interaction is invariant with respect to charge conjugation ${ }^{1}$. Therefore, to assume the existence of charge conjugation is physically well-motivated. The point here is that charge conjugation comes within the bosonic sector of the Standard Model since spontaneous symmetry breaking not only creates massive but also charged bosons ${ }^{2}$.

Of course, the decomposition (25) is the geometrical analogy to the usual complex linear combination of the electroweak bosons found in the literature on the Standard Model (see, eq. 5). Like in the local description the global decomposition (25) is unique, i. e. the correspondence between $\left(\xi_{W^{+}}, \xi_{W^{+}}\right)$and $\left(\xi_{\mathrm{w}_{1}}, \xi_{\mathrm{w}_{2}}\right)$ is one-to-one. Indeed, we have the following

Lemma 3.1 On the complex line bundle $\xi_{\mathrm{w}}$ there exists a complex conjugation iff it is trivial.

Proof: The statement follows from the fact that a complex vector bundle $\xi$ of rank N possesses a complex conjugation (i. e. a real structure) iff all of its odd Chern classes

\footnotetext{
${ }^{1}$ That is, the electromagnetic interaction does not permit to absolutely distinguish between particles and anti-particles.

${ }^{2}$ By "charge" we always mean "electromagnetic charge". We carefully distinguish between the notions of "charge" and "gauge coupling constant". The former is a dynamically conserved quantity due to Noether's theorem, whereas the latter is conserved by construction (it simply parameterizes the most general Killing form on $\operatorname{Lie}(\mathrm{G}))$.
} 
$c_{2 \mathrm{k}+1}(\xi) \in \mathrm{H}_{\mathrm{deR}}^{4 k+2}(\mathcal{M})$ vanish. Though this is hard to prove in general, for $\mathrm{N}=1$ the proof is elementary. Indeed, let $\xi_{\mathrm{w}}$ be trivial. Then, the structure group can be reduced to the identity and $\xi_{\mathrm{w}}$ possesses a canonical complex conjugation. If we let the complex line bundle $\xi_{\mathrm{w}}$ be equipped with a complex conjugation, $\xi_{\mathrm{w}}$ is the complexification of a real line bundle. When considered as a real vector bundle $\xi_{\mathrm{w}}$ decomposes into the Whitney sum of two real line bundles. However, since all one-dimensional representations of $\mathrm{SO}(2)$ are trivial it follows that each of the real line bundles is trivial.

As a consequence we conclude that with respect to a bosonic vacuum $(\Theta, \mathcal{V})$ the Yang-Mills bundle of the electroweak interaction reads ${ }^{3}$

$$
\xi_{\mathrm{YM}} \simeq \bigoplus^{4} \tau_{\mathrm{M}}^{*}
$$

Next, we show that this fully fixes the topological structure of both the electromagnetic and the electroweak gauge bundle.

Proposition 3.2 The gauge bundles underlying the electroweak interaction and electromagnetism are trivial.

Proof: Since $\xi_{\mathrm{w}}$ is a complex line bundle carrying the fundamental representation of the electromagnetic gauge group its frame bundle can be canonically identified with $\mathcal{Q}$. Since there exists a complex conjugation on $\xi_{\mathrm{w}}$ iff $\xi_{\mathrm{w}}$ is trivial, the principal $\mathrm{H} \equiv \mathrm{U}_{\text {elm }}(1)$-bundle $\mathcal{Q}$ must also be trivial ${ }^{4}$. Consequently, as an extension of the electromagnetic gauge bundle, the principal $\mathrm{SU}(2) \times \mathrm{U}(1)$ bundle $\mathcal{P}$ must also be trivial (see our corresponding remark of the last section).

As a consequence, we conclude that the $W^{ \pm}$-vector bosons of the weak interaction are charge conjugate to each other iff the electroweak gauge bundle is trivial. In what follows we will slightly change our argument and present two alternative proofs of the

\footnotetext{
${ }^{3}$ Because of the tensor product with the cotangent bundle, the Yang-Mills bundle $\xi_{\text {YM }}$ is always considered as a real vector bundle. Thus, to consider the Yang-Mills bundle as the tensor product of $\tau_{\mathrm{M}}^{*}$ with $(22)$ would not make sense.

${ }^{4}$ Here, the structure group of electromagnetism is defined by

$$
\mathrm{U}_{\text {elm }}(1) \simeq \mathrm{I}\left(\mathbf{z}_{0}\right)=\left\{h \equiv \exp (\theta[\mathrm{T}+\mathrm{y} i]) \mid \mathrm{T}=\mathrm{T}\left(\mathbf{z}_{0}\right) \in \operatorname{su}(2), \operatorname{tr}\left([\mathrm{T}+\mathrm{y} i]^{2}\right)=-1, \theta \in \mathbb{R}\right\} .
$$
}


triviality of the electroweak gauge bundle. The first proof refers to the existence of a bosonic vacuum $(\Theta, \mathcal{V})$. In contrast, the second proof only refers to the existence of a non-trivial ground state $\mathcal{V} \in \Gamma\left(\xi_{\text {orb }}\right)$ of the Higgs boson.

Proposition 3.3 The electroweak interaction admits a bosonic vacuum iff its underlying gauge bundle $\mathcal{P}$ is trivial.

Proof: Of course, if the principal $\mathrm{SU}(2) \times \mathrm{U}(1)$ bundle $\mathcal{P}$ is assumed to be trivial, then the YMH pair $\left(\Theta_{0}, \mathcal{V}_{0}\right)$ will serve as a vacuum for all minima $\mathbf{z}_{0} \in \mathbb{C}^{2}$. Now, let $(\Theta, \mathcal{V})$ be a vacuum with respect to the data defining the electroweak interaction as a $\mathrm{YMH}$ gauge theory. Then, a principal $\mathrm{U}_{\text {elm }}(1)$-bundle $\mathcal{Q}$ together with a bundle embedding $\iota: \mathcal{Q} \hookrightarrow \mathcal{P}$ uniquely corresponds to $\mathcal{V}$ such that $\iota^{*} \Theta$ is also a flat connection on the reduced bundle $\mathcal{Q}$. Thus, the first Chern class $c_{1}(\mathcal{Q}) \in \mathrm{H}_{\mathrm{deR}}^{2}(\mathcal{M})$ of the electromagnetic gauge bundle must vanish. Since principal U(1)-bundles are classified by their first Chern class it follows that $\mathcal{Q}$ must be trivial. Since the electromagnetic gauge bundle $\mathcal{Q}$ is regarded as a reduction of the electroweak gauge bundle $\mathcal{P}$, the latter must be also trivial.

Like in the case of the assumption $\pi_{1}(\mathcal{M})=0$ the above given argument makes use of the existence of a flat connection $\Theta$ on $\mathcal{P}$. While physically motivated, this assumption turns out to be mathematically very restrictive. However, taking charge conjugation into account, we may draw the same conclusion as above by only referring to the existence of a non-trivial ground state of the Higgs boson.

Proposition 3.4 The Higgs boson of the electroweak sector of the Standard Model possesses a non-trivial ground state iff the electroweak interaction is geometrically modeled by the trivial principal $\mathrm{SU}(2) \times \mathrm{U}(1)$ bundle.

Proof: Again, if the electroweak gauge bundle $\mathcal{P}$ is supposed to be trivial, then every minimum $\mathbf{z}_{0} \in \mathbb{C}^{2}$ gives rise to a canonical section of the (also trivial) orbit bundle. To prove the converse, let $\mathcal{V} \in \Gamma\left(\xi_{\text {orb }}\right)$ be a section of the orbit bundle with respect to some chosen minimum $\mathbf{z}_{0} \in \mathrm{S}^{3}$. Also, let $(\mathcal{Q}, \iota)$ be the corresponding electromagnetic reduction of the electroweak gauge bundle $\mathcal{P}$. Again, the isomorphism class of the electromagnetic gauge bundle $\mathcal{Q}$ is fully determined by its first Chern class $c_{1}(\mathcal{Q}) \in \mathrm{H}_{\mathrm{deR}}^{2}(\mathcal{M})$ 
(c.f., for instance, the appendix of [Free/Uhle'84]). Therefore, electromagnetism is invariant with respect to charge conjugation iff $c_{1}(\mathcal{Q})=0$.

Even though it only refers to the existence of a non-trivial ground state of the Higgs boson the above result also implies, of course, the existence of a bosonic vacuum as, for instance, the canonical one represented by the YMH pair $\left(\Theta_{0}, \mathcal{V}_{0}\right)$. Our main theorem then says that in the case of the electroweak interaction this kind of a bosonic vacuum is in fact the only one, provided that $\mathrm{H}_{\mathrm{deR}}^{1}(\mathcal{M})=0$. The proof of this statement makes use of another special feature of the electroweak interaction.

Theorem 3.1 Let $\left(\mathcal{P}, \rho_{\mathrm{H}}, \mathrm{V}_{\mathrm{H}}\right)$ be the data defining the electroweak interaction as a $Y M H$ gauge theory. Then, the corresponding moduli space of bosonic vacua $\mathfrak{M}_{\text {vac }}$ is an affine space with vector space $\mathrm{H}_{\mathrm{deR}}^{1}(\mathcal{M})$.

Proof: According to the above propositions we already know that the moduli space of bosonic vacua is non-empty iff the electroweak gauge bundle $\mathcal{P}$ is trivial. As a consequence, every vacuum section $\mathcal{V}$ can be identified with a smooth mapping $\nu: \mathcal{M} \rightarrow \operatorname{orbit}\left(\mathbf{z}_{0}\right) . \quad$ In [Tolk'03(a)] it was shown, however, that in the case of the electroweak interaction the principal H-bundle

$$
\begin{aligned}
\mathrm{G} & \longrightarrow \operatorname{orbit}\left(\mathbf{z}_{0}\right) \\
g & \mapsto g \mathbf{z}_{0}
\end{aligned}
$$

is also trivial. Therefore, every smooth mapping $\nu$ possesses a smooth lift $\gamma: \mathcal{M} \rightarrow \mathrm{G}$, such that $\nu(x)=\gamma(x) \mathbf{z}_{0}$. In other words, every vacuum section is gauge equivalent to the canonical vacuum section. Moreover, since the affine space of H-reducible connections $\mathcal{A}$ on $\mathcal{P}$ can be canonically identified with $\Omega^{1}(\mathcal{M})$ it follows that each flat connection $\Theta$ uniquely corresponds to an element of $\mathrm{H}_{\mathrm{deR}}^{1}(\mathcal{M})$. If the latter is trivial, then $\Theta$ is gauge equivalent to the canonical connection $\Theta_{0}$ and the moduli space of bosonic vacua consists of at most one point represented by the canonical Yang-MillsHiggs pair $\left(\Theta_{0}, \mathcal{V}_{0}\right)$.

We may thus summarize our main result by

$$
\mathfrak{M}_{\text {vac }} \simeq \mathrm{H}_{\text {deR }}^{1}(\mathcal{M}),
$$


iff the electroweak gauge bundle $\mathcal{P}$ is trivial. This in turn is in one-to-one correspondence with the assumption of the existence of charge conjugation $\mathcal{J}$. Locally, the relation (5) between the "interacting fields" $(W, B, \Phi)$ and the "(asymptotically) free fields" ( $\left.A_{\text {elm }}, Z^{0}, W^{ \pm}, \Phi_{\mathrm{H}, \text { phys }}\right)$ is unambiguous. However, whether this holds also true when seen from a global perspective depends on the structure of the moduli space $\mathfrak{M}_{\text {vac }}$ of electroweak vacua.

Let $\Delta: \mathcal{M} \rightarrow \mathcal{M} \times \mathcal{M}$ be the diagonal embedding $x \mapsto(x, x)$. Since the structure group $\mathrm{G} \equiv \mathrm{SU}(2) \times \mathrm{U}(1)$ of the electroweak gauge bundle $\mathcal{P}$ is a direct product one obtains $^{5}$

$$
\mathcal{P}=\Delta^{*}\left(\mathcal{P}_{2} \times \mathcal{P}_{1}\right) .
$$

Here, respectively, $\mathcal{P}_{1}$ and $\mathcal{P}_{2}$ are appropriate principal U(1) and principal $\mathrm{SU}(2)$ bundles over $\mathcal{M}$, and $\Delta^{*}\left(\mathcal{P}_{2} \times \mathcal{P}_{1}\right)$ means the pull-back bundle of $\mathcal{P}_{2} \times \mathcal{P}_{1}$ with respect to $\Delta$. According to the Higgs dinner (17) with respect to an electroweak vacuum $(\Theta, \mathcal{V}) \in \mathcal{A}\left(\xi_{\mathrm{H}}\right) \times \Gamma\left(\xi_{\mathrm{H}}\right)$, we obtain the following two orthogonal decompositions of the adjoint bundle $\mathfrak{a r}(\mathcal{P})$ (and thus of the Yang-Mills bundle $\xi_{\mathrm{YM}}$ )

$$
\begin{aligned}
\mathfrak{a} \mathfrak{d}(\mathcal{P}) & =\mathfrak{a} \mathfrak{d}\left(\mathcal{P}_{1}\right) \oplus \mathfrak{a} \mathfrak{d}\left(\mathcal{P}_{2}\right) \\
& \simeq \mathfrak{a} \mathfrak{d}(\mathcal{Q}) \oplus \xi_{\mathrm{z}} \oplus \xi_{\mathrm{w}} .
\end{aligned}
$$

Because of $\xi_{\mathrm{w}} \subset \mathfrak{a} \mathfrak{d}\left(\mathcal{P}_{2}\right)$, there is a unique real line bundle $\xi_{\mathrm{w}_{3}} \subset \mathfrak{a} \mathfrak{d}\left(\mathcal{P}_{2}\right)$, such that

$$
\mathfrak{a} \mathfrak{d}\left(\mathcal{P}_{2}\right) \simeq \xi_{\mathrm{W}} \oplus \xi_{\mathrm{w}_{3}} .
$$

The "electroweak mixing angle" $\theta_{\mathrm{w}}$ of $(5)$ is geometrically represented by the isometric isomorphism (over the identity on $\mathcal{M}$ )

$$
\mathfrak{a} \mathfrak{d}\left(\mathcal{P}_{1}\right) \oplus \xi_{\mathrm{w}_{3}} \simeq \mathfrak{a} \mathfrak{d}(\mathcal{Q}) \oplus \xi_{\mathrm{z}} .
$$

Note that both sides of (32) are orthogonal complements of $\xi_{\mathrm{w}} \subset \mathfrak{a} \mathfrak{d}(\mathcal{P})$. Also note that the isomorphism (32) does not take into account the triviality of the electroweak gauge bundle. The triviality of $\mathcal{P}$ only corresponds to the last relation of (5) which is geometrically described by (25). Of course, the isomorphism (32) only depends on $[(\Theta, \mathcal{V})] \in \mathfrak{M}_{\text {vac }}$.

\footnotetext{
${ }^{5}$ I would like to thank E. Binz for a corresponding hint.
} 
If space-time is assumed to be simply connected, then, up to gauge equivalence, there is at most one non-trivial bosonic vacuum. This, of course, holds true for $\left(\mathcal{M}, g_{\mathrm{M}}\right) \simeq \mathbb{R}^{1,3}$, usually encountered in particle physics. This also fits in with the corresponding results presented in [Tolk'03(a)] for general YMH data $\left(\mathcal{P}, \rho_{\mathrm{H}}, V_{\mathrm{H}}\right)$, where, we showed that $\pi_{1}(\mathcal{M})=0$ implies that the moduli space of bosonic vacua consists of at most one point. In the case considered in the present paper, however, the topology of space-time is two-fold related to the bosonic vacua; the existence is tied to $\mathrm{H}_{\mathrm{deR}}^{2}(\mathcal{M})$, whereas uniqueness is related to $\mathrm{H}_{\mathrm{deR}}^{1}(\mathcal{M})$. The non-triviality of the latter may physically be interpreted in terms of the Ahoronov-Bohm effect. The anholonomy of the electron's phase uncovers the non-triviality of the electroweak vacuum.

\section{Asymptotically Free Particles}

In this section we summarize the geometrical meaning of the physical notion of a "free particle" in the realm of gauge theory. The motivation for this is as follows (see also our introduction): On the one hand the notion of a free particle seems basic for the interpretation of "mass" and "charge" of an elementary particle. Also, in the case of perturbation theory this notion is crucial. On the other hand, the notion of "freeness" in this context refers to "non-interaction" and thus seems to contradict the dogma of gauge independence. However, the geometrical description of spontaneously broken gauge theories presented here permits to also describe the notion of a "free particle" in purely geometrical terms. Thus, the spontaneous symmetry breaking of the electroweak interaction permits the geometrical combination of the notion of a "free particle" with its "mass" and its "charge".

For this let $t \in[0,1]$. A one-parameter family of Yang-Mills-Higgs pairs $\left(\mathcal{A}_{t}, \Phi_{t}\right) \in$ $\mathcal{A}\left(\xi_{\mathrm{H}}\right) \times \Gamma\left(\xi_{\mathrm{H}}\right)$ is called a "linear fluctuation" of a bosonic vacuum (pair) $(\Theta, \mathcal{V})$ provided that

$$
\begin{aligned}
\mathcal{A}_{t} & =\Theta+t(\mathcal{A}-\Theta), \\
\Phi_{t} & =\mathcal{V}+t \Phi
\end{aligned}
$$

Here, $\mathcal{A} \in \mathcal{A}\left(\xi_{\mathrm{H}}\right)$ is a connection associated with a principal connection on $\mathcal{P}$ which is non-compatible with the vacuum; $\Phi \in \Gamma\left(\xi_{\mathrm{H}}\right)$ is a section in the unitary gauge ${ }^{6}$, i. e. $\Phi$

\footnotetext{
${ }^{6}$ Despite the terminology used the unitary "gauge" is in fact not a choice of gauge. Instead it refers to a specific choice of vacuum section adapted to the section $\Phi$ under consideration (see [Tolk'03(a)]).
} 
uniquely corresponds to a section $\Phi_{\mathrm{H} \text {,phys }} \in \Gamma\left(\xi_{\mathrm{H}, \mathrm{phys}}\right)$. Of course, the definition (33) is the geometrical analogy to (1).

With respect to $(\Theta, \mathcal{V})$ the connection $\mathcal{A}$ uniquely corresponds to a section $A \in$ $\Gamma\left(\xi_{\mathrm{YM}}\right)$. Moreover, because of the decomposition (26) we have

$$
A=A_{\mathrm{elm}}+Z^{0}+W^{ \pm}
$$

where $A_{\text {elm }} \in \Gamma\left(\xi_{\text {elm }}\right) \simeq \Omega^{1}(\mathcal{M}), Z^{0} \in \Gamma\left(\xi_{\mathrm{z}^{0}}\right) \simeq \Omega^{1}(\mathcal{M})$ and $W^{ \pm} \in \Gamma\left(\xi_{\mathrm{w}^{ \pm}}\right) \simeq \Omega^{1}(\mathcal{M})$.

The Euler-Lagrange equations of the Yang-Mills-Higgs functional $\mathcal{I}_{\text {YMH }}$ with respect to the fluctuation $\left(\mathcal{A}_{t}, \Phi_{t}\right)$ up to $\mathcal{O}(t)$ yield the well-known "free field equations"

$$
\begin{aligned}
\delta d A_{\text {elm }} & =0, \\
\delta d Z^{0}+\mathrm{m}_{\mathrm{z}}^{2} Z^{0} & =0, \\
\delta d W^{ \pm}+\mathrm{m}_{\mathrm{W}}^{2} W^{ \pm} & =0, \\
\delta d \Phi_{\mathrm{H}, \text { phys }}+\mathrm{m}_{\mathrm{H}, \text { phy }}^{2} \Phi_{\mathrm{H}, \text { phys }} & =0 .
\end{aligned}
$$

Note that these second order equations are indeed $\mathrm{H}$-invariant (but not G-invariant) and that $\delta Z^{0}=\delta W^{ \pm}=\delta \Phi_{\mathrm{H} \text {,phys }}=0$ for reasons of consistency. Here, " $d$ " denotes the exterior covariant derivative with respect to the trivial connection, " $\delta$ " its covariant co-derivative and $\mathrm{m}_{\mathrm{H}, \mathrm{phy}} \in \operatorname{spec}\left(\mathcal{V}^{*} \mathrm{M}_{\mathrm{H}}^{2}\right)$ the "mass" of the physical Higgs boson", where $\mathcal{V}^{*} \mathrm{M}_{\mathrm{H}}^{2} \in \Gamma\left(\operatorname{End}\left(\mathrm{E}_{\mathrm{H}}\right)\right)$ is the Higgs mass matrix (c.f. [Tolk'03(a)]). Also note that

$$
\delta d W^{+}+\mathrm{m}_{\mathrm{W}}^{2} W^{+}=\mathcal{J}\left(\delta d W^{-}+\mathrm{m}_{\mathrm{w}}^{2} W^{-}\right) .
$$

Since, with respect to any geodesic coordinate system (local "inertial system"), the principal symbols $\sigma_{\mathrm{pr}}$ of the above second order differential operators asymptotically $(t \rightarrow 0)$ equals the total symbols $\sigma$, one may consider the above sections (34) and $\Phi=\Phi_{\mathrm{H} \text {,phys }}$ as geometrically representing states of asymptotically free particles (in semi-classical approximation) that are represented by the corresponding (trivial) line bundles. Let $\square:=\delta d+d \delta$ be the covariant wave operator ("d'Alambert operator") with respect to the canonical connections on the trivial line bundles representing, respectively, the photon $\xi_{\text {elm }}$ the massive and (un-)charged vector bosons of the weak interaction, $\xi_{\mathrm{z}^{0}}, \xi_{\mathrm{w}^{ \pm}}$, and the physical Higgs boson $\xi_{\mathrm{H} \text {,phys }}$. Also let $\mathcal{C}^{+}(\mathcal{M}) \subset \mathrm{T}^{*} \mathcal{M}$ be (pointwise) the future oriented part of the light cone that is defined by the Lorentz

\footnotetext{
${ }^{7}$ In the case of rotationally symmetric Higgs potentials the rank of the physical Higgs bundle equals one, which equals the rank of the Higgs mass matrix.
} 
structure $g_{\mathrm{M}}$. Then, for all $\xi \in \mathcal{C}^{+}(\mathcal{M})$ the free field equations (35) indeed imply the well-known dispersion relation between mass, energy and momentum of a noninteracting pointlike particle

$$
\sigma(-\square)(\xi) \stackrel{*}{=} \sigma_{\mathrm{pr}}(-\square)(\xi)=g_{\mathrm{M}}(\xi, \xi)=\left\{\begin{array}{rll}
0 & : & \xi_{\text {elm }} \\
\mathrm{m}_{\mathrm{Z}^{0}}^{2}, \mathrm{~m}_{\mathrm{W}}^{2} & : & \xi_{\mathrm{Z}^{0}}, \xi_{\mathrm{W}^{ \pm}} \\
\mathrm{m}_{\mathrm{H}, \mathrm{phys}}^{2} & : & \xi_{\mathrm{H}, \mathrm{phys}}
\end{array}\right.
$$

Here, "*" means "with respect to any geodesic coordinate system".

While the local form of (35) and (37) can be found in almost any text book of quantum field theory we summarized them here to put emphasis on their geometrical content. In fact, the first equality of the relations (37) might serve as a geometrical definition of "freeness", the second equality combines the notions of particle and of field and the third equality indicates the geometrical background of the former two equalities in the realm of (spontaneously broken) gauge theories. Notice that the right hand side of the dispersion relation (37) is fully determined by the sections $\mathcal{V}^{*} \mathrm{M}_{\mathrm{YM}}^{2} \in \Gamma(\operatorname{End}(\operatorname{ad}(\mathrm{P}))), \mathcal{V}^{*} \mathrm{M}_{\mathrm{H}}^{2} \in \Gamma\left(\operatorname{End}\left(\mathrm{E}_{\mathrm{H}}\right)\right.$ together with the assumption that electromagnetism is invariant with respect to charge conjugation. That the spectrum of these sections is well-defined (i. e. constant and independent of the vacuum section chosen) is part of the mathematical structure of spontaneously broken gauge theories. The appropriate physical interpretation of the spectrum, however, is motivated only by the first two equalities on the left hand side of (37).

We close this section with a discussion on the geometrical meaning of the physical particles (6) of the electroweak interaction. For this we consider the Higgs bundle $\xi_{\mathrm{H}}$ as a real vector bundle of rank four. Each YMH pair $(\mathcal{A}, \Phi)$ yields a specific embedding of $\mathcal{M}$ into the total space $\mathrm{E}_{\mathrm{H}}$ of the Higgs bundle and a specific splitting of the tangent bundle $\tau_{\mathrm{H}}$ of $\mathrm{E}_{\mathrm{H}}$ into its horizontal and vertical part

$$
\tau_{\mathrm{H}}=\mathfrak{h}_{\mathrm{H}} \oplus \mathfrak{v}_{\mathrm{H}} .
$$

Moreover, $\left(g_{\mathrm{M}}, \mathcal{A}\right)$ turns $\mathrm{E}_{\mathrm{H}}$ into a (semi-)Riemannian manifold of $\operatorname{dimension} 4 \operatorname{dim} \mathcal{M}$ such that the splitting (38) becomes orthogonal.

Let $(\Theta, \mathcal{V})$ be an electroweak vacuum and $(\mathcal{A}, \Phi)$ be a (linear) fluctuation thereof. We call $\mathcal{M}_{\text {phys }}:=\mathcal{V}(\mathcal{M})$ the "physical space-time" with respect to the vacuum $(\Theta, \mathcal{V})$. Accordingly, we denote by $g_{\mathrm{H}}$ the (pseudo) metric on $\mathrm{E}_{\mathrm{H}}$ with respect to $\left(g_{\mathrm{M}}, \Theta\right)$. Be- 
cause of $\xi_{\text {orb }} \subset \xi_{\mathrm{H}}$ one obtains the following orthogonal decompositions along $\mathcal{M}_{\text {phys }}$ :

$$
\begin{aligned}
\left.\tau_{\mathrm{H}}\right|_{\mathrm{M}, \text { phys }} & \left.\left.\simeq \tau_{\mathrm{Orb}}\right|_{\mathrm{M}, \text { phys }} \oplus \nu_{\mathrm{Orb}}\right|_{\mathrm{M}, \text { phys }} \\
& \left.\left.\left.\simeq \mathfrak{h}_{\mathrm{Orb}}\right|_{\mathrm{M}, \text { phys }} \oplus \pi_{\mathrm{H}}^{*} \xi_{\mathrm{G}}\right|_{\mathrm{M}, \text { phys }} \oplus \pi_{\mathrm{H}}^{*} \xi_{\mathrm{H}, \text { hhys }}\right|_{\mathrm{M}, \text { phys }} \\
& \left.\left.\left.\left.\simeq \tau_{\mathrm{M}, \text { phys }} \oplus \pi_{\mathrm{H}}^{*} \xi_{\mathrm{Z}}\right|_{\mathrm{M}, \text { phys }} \oplus \pi_{\mathrm{H}}^{*} \xi_{\mathrm{W}}\right|_{\mathrm{M}, \text { phys }} \oplus \pi_{\mathrm{H}}^{*} \xi_{\mathrm{W}_{2}}\right|_{\mathrm{M}, \text { phys }} \oplus \pi_{\mathrm{H}}^{*} \xi_{\mathrm{H}, \text { phys }}\right|_{\mathrm{M}, \text { phys }} .
\end{aligned}
$$

Here, respectively, $\tau_{\mathrm{Orb}} a n d \nu_{\mathrm{Orb}}$ denote the tangent and the normal bundle of the orbit bundle and $\tau_{\mathrm{M} \text {,phys }}$ is the tangent bundle of the physical space-time.

Correspondingly, for every $w=(\mathcal{V}(x), \mathbf{w}) \in \mathrm{TE}_{\mathrm{H}}$ there are real constants $\lambda_{\mathrm{elm}}, \lambda_{0}$, $\lambda_{1}, \lambda_{2}$ and $\lambda_{\mathrm{H}, \text { phys }}$ such that $w$ can be written in terms of the solutions of the free field equations (35):

$$
w=\lambda_{\text {elm }} \mathrm{T}\left(\mathcal{V} \circ \pi_{\mathrm{H}}\right)(w)+\lambda_{0} \mathbf{Z}^{0}(w)+\lambda_{1} \mathbf{W}^{1}(w)+\lambda_{2} \mathbf{W}^{2}(w)+\lambda_{\mathrm{H}, \text { phys }} \Phi_{\mathrm{H}, \text { phys }}(x) .
$$

Here, for instance, $\left.\left.\mathbf{Z}^{0}(w) \equiv \rho_{\mathrm{H}}^{\prime}\left(\pi_{\mathrm{H}}^{*} Z^{0}(w)\right) \mathcal{V} \in \mathrm{TE}\right|_{\mathrm{H}}\right|_{\mathrm{M} \text {,phys }}$ etc., and $\rho_{\mathrm{H}}^{\prime}:=\mathrm{d} \rho_{\mathrm{H}}(e)$ is the (real form of the) induced representation on $\operatorname{Lie}(\mathrm{G})$. Notice that we have made use of the Higgs dinner (17) in such a way that $\pi_{\mathrm{H}}^{*} Z^{0} \in \Omega^{1}\left(\mathrm{E}_{\mathrm{H}}, \operatorname{ad}(\mathrm{P})\right)$ and that the gauge group $\mathcal{G}$ of the electroweak gauge bundle naturally acts from the right on the orbit bundle.

As was shown in [Tolk'03(a)] the restriction to $\mathcal{M}_{\text {phys }}$ of any compatible connection $\mathcal{A}_{\text {elm }}$ on $\xi_{\mathrm{H}}$ coincides with the canonical "connection" that is defined by the vacuum section $\mathcal{V}$. That is, if we denote by $\wp_{\mathrm{elm}}^{\mathrm{H}}$ the horizontal projector on $\tau_{\mathrm{H}}$ with respect to the connection $\mathcal{A}_{\text {elm }}$ then

$$
\left.\wp_{\mathrm{elm}}^{\mathrm{H}}\right|_{\mathcal{V}(x)}(\mathbf{w})=\mathrm{d} \mathcal{V}(x)\left(\mathrm{d} \pi_{\mathrm{H}}(\mathcal{V}(x)) \mathbf{w}\right),
$$

for all $x \in \mathcal{M}$ and $\mathbf{w} \in \mathrm{T}_{\mathcal{V}(x)} \mathrm{E}_{\mathrm{H}}$. Moreover, with respect to the decomposition (34) any connection $\mathcal{A}$ on the Higgs bundle can be written as

$$
\begin{aligned}
\mathcal{A} & :=\Theta+\mathbf{A} \\
& =\mathcal{A}_{\text {elm }}+\mathbf{A}_{\mathrm{G}} .
\end{aligned}
$$

Here, $\mathcal{A}_{\text {elm }}=\Theta+\mathbf{A}_{\text {elm }}$ and $\mathbf{A}_{\mathrm{G}} \equiv \mathbf{Z}^{0}+\mathbf{W}^{1}+\mathbf{W}^{2}$. Then, the horizontal projector with respect to an arbitrary (associated) connection $\mathcal{A} \in \Gamma\left(\xi_{\mathrm{H}}\right)$ reads

$$
\wp_{\mathrm{A}}^{\mathrm{H}}=\wp_{\mathrm{elm}}^{\mathrm{H}}+\mathbf{A}_{\mathrm{G}}
$$


and thus

$$
\left.\wp_{\mathrm{A}}^{\mathrm{H}}\right|_{\mathrm{M}, \text { phys }}(w)=\mathrm{T}\left(\mathcal{V} \circ \pi_{\mathrm{H}}\right)(w)+\mathbf{A}_{\mathrm{G}}(w)
$$

for all $w=(\mathcal{V}(x), \mathbf{w}) \in \mathrm{TE}_{\mathrm{H}}$.

Since the decomposition (39) is orthogonal with respect to $g_{\mathrm{H}}$ one obtains for all $w_{i}=\left(\mathcal{V}(x), \mathbf{w}_{i}\right) \in \mathrm{TE}_{\mathrm{H}}(i=1,2)$

$$
\begin{aligned}
\left.g_{\mathrm{H}}^{\mathrm{A}}\right|_{\mathrm{M}, \text { phys }}\left(w_{1}, w_{2}\right) & :=\kappa_{\mathrm{H}}\left(\left.\wp_{\mathrm{A}}^{\mathrm{V}}\right|_{\mathrm{M}, \text { phys }}\left(w_{1}\right),\left.\wp_{\mathrm{A}}^{\mathrm{V}}\right|_{\mathrm{M}, \text { phys }}\left(w_{2}\right)\right)+\pi_{\mathrm{H}}^{*} g_{\mathrm{M}}\left(w_{1}, w_{2}\right) \\
& =\left.g_{\mathrm{H}}\right|_{\mathrm{M}, \text { phys }}\left(w_{1}, w_{2}\right)+\kappa_{\mathrm{H}}\left(\mathbf{A}_{\mathrm{G}}\left(w_{1}\right), \mathbf{A}_{\mathrm{G}}\left(w_{2}\right)\right),
\end{aligned}
$$

where, respectively, $\wp_{\mathrm{A}}^{\mathrm{V}}$ is the vertical projector that is defined by (43) and $\kappa_{\mathrm{H}}$ is the Hermitian form on the Higgs bundle.

The relation (45) shows that the massive weak vector bosons $\left(Z^{0}, W^{ \pm}\right)$correspond to normal sections of $\mathcal{M}_{\text {phys }}$ which yield a "fluctuation" of the (pseudo) metric $g_{\mathrm{H}}$. In contrast, the massless photon $A_{\text {elm }}$ only gives rise to a change of $g_{\mathrm{H}}$ off the physical space-time $\mathcal{M}_{\text {phys }}$. Note that, when restricted to $\mathcal{M}_{\text {phys }}$, the connection $\mathcal{A}_{\text {elm }}$ is flat. In particular, one obtains $d_{\mathrm{A}, \mathrm{elm}} \mathcal{V}=0$ and thus

$$
d_{\mathrm{A}} \mathcal{V}=\mathbf{A}_{\mathrm{G}} .
$$

We stress that it is this relation between an arbitrary connection on the electroweak gauge bundle $\mathcal{P}$ and the electroweak vacuum that yields a non-trivial Yang-Mills mass matrix (18).

\section{Remarks}

In the following we give some comments on the results presented.

The presented classification theorem concerning the bosonic vacua in the case of the electroweak interaction can be generalized to more general YMH data $\left(\mathcal{P}, \rho_{\mathrm{H}}, \mathrm{V}_{\mathrm{H}}\right)$ whereby the "little group" $\mathrm{H}$ is supposed to be given either by $\mathrm{U}(1)$, or by $\mathrm{SU}(2)$. The general Higgs potential is, again, assumed to be rotationally symmetric. 
In the slightly more general case with $\mathrm{H}=\mathrm{U}(1)$, the moduli space of bosonic vacua can be identified with

$$
\mathfrak{M}_{\mathrm{vac}}=\mathrm{H}_{\mathrm{deR}}^{1}(\mathcal{M}) \times \# \mathrm{Orb}
$$

where \#Orb denotes the number of orbits.

For instance, in the case of a "Sine-Gordon"-like Higgs potential

$$
\mathrm{V}_{\mathrm{H}}(\mathbf{z}):=\frac{\mu^{2}}{\lambda^{2}}(1-\cos (\lambda|\mathbf{z}|)) \quad(\mu, \lambda>0)
$$

the moduli space of bosonic vacua reads

$$
\mathfrak{M}_{\mathrm{vac}}=\mathrm{H}_{\mathrm{deR}}^{1}(\mathcal{M}) \times \mathbb{Z}
$$

In the case where the isotropy group of a minimum $\mathbf{z}_{0}$ of a general Higgs potential can be identified with $\mathrm{SU}(2)$ one obtains

$$
\mathfrak{M}_{\mathrm{vac}}=\mathfrak{M} \times \# \text { Orb }
$$

where $\mathfrak{M}$ is the moduli space of flat $\mathrm{SU}(2)$-connections on the trivial principal $\mathrm{SU}(2)$ bundle over space-time $\mathcal{M}$.

The results presented imply that the set of non-vanishing sections of the Higgs bundle is in one-to-one correspondence with the non-vanishing smooth real-valued functions on space-time $\mathcal{M}$, i. e.

$$
\Gamma^{*}\left(\xi_{\mathrm{H}}\right) \equiv \Gamma\left(\xi_{\mathrm{H}}\right) \backslash\{\mathcal{O}\} \simeq \mathcal{C}^{\infty}(\mathcal{M}) \backslash\{0\}
$$

where $\mathcal{O}$ (resp. 0) is the zero section (resp. zero function) on $\mathcal{M}$. From theorem (3.1) it follows that the Higgs bundle is trivial and therefore $\Gamma\left(\xi_{\mathrm{H}}\right) \simeq \Omega^{0}\left(\mathcal{M}, \mathbb{C}^{2}\right)$. If one identifies $\mathbb{C}^{2} \simeq \mathbb{H}$ with the quaternions via $\mathbf{z}=\left(z_{1}, z_{2}\right) \mapsto q=z_{1}+z_{2} j$, then one may make use of the polar decomposition for non-zero quaternions $q=\|q\| \exp (\vartheta n)$ $\left(n \in \mathbb{H}, n^{2}=-1\right)$ to show that $\Phi \in \Gamma^{*}\left(\xi_{\mathrm{H}}\right)$ is gauge equivalent to the mapping

$$
\begin{aligned}
\mathcal{M} & \longrightarrow \mathrm{E}_{\mathrm{H}} \\
x & \mapsto\left(x,\|\Phi(x)\| \mathbf{e}_{0}\right)
\end{aligned}
$$

with $\mathbf{e}_{0}:=\mathbf{z}_{0} /\left\|\mathbf{z}_{0}\right\| \in S^{3}$ 
Accordingly, a section of the physical Higgs bundle $\xi_{\mathrm{H}, \mathrm{phys}}$ reads

$$
\begin{aligned}
\Phi_{\mathrm{H}, \text { phys }}: \mathcal{M} & \longrightarrow \mathrm{E}_{\mathrm{H}, \text { phys }} \\
x & \mapsto(x,\|\Phi(x)\|),
\end{aligned}
$$

where we have identified $\mathbb{R}^{4} \supset \mathrm{W}_{\mathrm{H}, \mathrm{phys}}:=\mathbb{R} \mathbf{e}_{0}$ with $\mathbb{R}$. Moreover, with help of this identification the free field equation for the physical Higgs boson is reduced to the ordinary Klein-Gordon equation for the function $\varphi:=\|\Phi\|$.

The mapping (52) is known in physics as the "Higgs boson in the unitary gauge". As is well-known, this terminology refers to the fact that the "phase" of a particle is unphysical and can be thus "gauged away". In fact, when considered as a field, $n \in \mathbb{R}^{3} \subset \mathbb{H}$ geometrically corresponds to a section of the Goldstone bundle $\xi_{\mathrm{G}}$. For the above given argument which is used in physics to show the existence of the unitary gauge in the electroweak interaction and which leads to (51) it seems crucial that the "phase of the Higgs boson" can be identified with an element of $\mathrm{SU}(2) \subset \mathrm{G}$. However, this turns out not to be the case, actually. Indeed, the isomorphism (51) only depends on the structure of the Higgs potential and not, e. g., of the simple structure of $\mathfrak{M}_{\text {vac }}$. In particular, the existence of the unitary gauge does not depend on the triviality of the Higgs bundle. For example, in contrast to the above given argument (which only works in the case where $\mathcal{P}$ is trivial), the isomorphism (51) always holds true for rotationally symmetric Higgs potentials. In this case, the physical Higgs bundle must be necessarily trivial for every vacuum section and thus also (53) generally holds true (c. f. [Tolk'03(a)]). For rotationally symmetric Higgs potentials the question about the existence of the unitary gauge is related to the question whether $\Gamma^{*}\left(\xi_{\mathrm{H}}\right)$ is empty or not. But this is basically the same as to ask about the existence of vacuum sections which spontaneously break the gauge symmetry. Therefore, the assumption that $\Gamma^{*}\left(\xi_{\mathrm{H}}\right) \neq \emptyset$ is physically well-motivated. The results presented here with respect to the electroweak interaction show that in the case of ordinary electromagnetism $\Gamma^{*}\left(\xi_{\mathrm{H}}\right) \neq \emptyset$ is the same as the triviality of the electroweak gauge bundle (see proposition (3.3)).

Although, in general, the notion of "free particles" depends on the gauge class $[(\Theta, \mathcal{V})] \in \mathfrak{M}_{\text {vac }}$ of electroweak vacua, the geometrical interpretation of the particle content of the electroweak interaction holds true for all vacua. Moreover, the relation (46) remains intact for arbitrary connections $\mathcal{A}_{\text {elm }}$. It only makes use of the compatibility condition (13) and the triviality (41) of $\mathcal{A}_{\text {elm }}$ along the physical space-time $\mathcal{M}_{\text {phys }}$. Notice that the latter geometrical property of an H-reducible connection guarantees that 
the spectrum of the bosonic mass matrices is constant. Accordingly, the Higgs dinner (17) also works in the case when the gauge bundle $\mathcal{P}$ possesses no flat connections. This remark becomes important, for example, when non-trivial U(1)-reductions (resp. $\mathrm{SU}(2)$-reductions) of $\mathcal{P}$ are considered. We close this section with the remark that the intrinsic geometry of the physical space-time $\mathcal{M}_{\text {phys }}$ is the same as that of the "naked" space-time $\mathcal{M}$. We summarize this by saying that in the case of ordinary electromagnetism the structure of the moduli space of the electroweak vacua $\mathfrak{M}_{\text {vac }}$ only depends on the topology of space-time but not on its geometry. This, however, may change for non-trivial vacua.

\section{Conclusion}

In this paper we have discussed the moduli space of bosonic vacua of the electroweak interaction on "tree level". We have proved that the corresponding moduli space is either empty or an affine space that can be canonically identified with the first de Rham cohomology group of space-time. We have shown that, when charge conjugation is taken into account, the existence of non-trivial ground states of the Higgs boson is equivalent to the triviality of the electroweak gauge bundle. For this, however, it is crucial that spontaneous symmetry breaking of the electroweak interaction not only yields massive but also electrically charged bosons. It follows that the electromagnetic gauge bundle must be also trivial. For this reason one may ask about the existence of magnetic monopoles within the realm of the Standard Model. Basically, there are two answers to this question: On the one hand, one may consider a magnetic monopole as a physical object in its own which is independent of the Standard Model. This, however, raises the question of the physical meaning of the underlying U(1) gauge bundle of the monopole and its relation to Dirac's quantization condition of electric charge. On the other hand, since a monopole is assumed to be massive and related to electromagnetism it seems far more natural to consider it as a possibly non-trivial electromagnetic reduction of the electroweak interaction. In this case, a monopole is not considered as a separate particle but as a certain ground state of the Higgs boson which is gauge inequivalent to the ground state usually encountered in perturbation theory (i. e. to $\mathcal{V}_{0}$ ). In any case, the existence of a monopole field would spoil the symmetry under charge conjugation. Since the latter has been shown in this paper to be intimately related to the structure of the moduli space of electroweak vacua, it 
is natural to complete our discussion on the geometrical structure of the electroweak interaction by considering the case where charge conjugation $\mathcal{J}$ is not assumed to exist. This will be done in a forthcoming paper.

\section{Acknowledgments}

I am very grateful to E. Binz for stimulating discussions on the subject matter discussed in this paper.

\section{References}

[Aitch/Hey'82] Aitchison, I. J. R. and Hey, A. J. G.: Gauge Theories in Particle Physics, Adam Hilger Ltd, Bristol (1982).

[Blee'81] Bleecker, D.: Gauge Theory and Variational Principles, Global Analysis, Addison-Wesley Pub. Co. (1981).

[Choq/deWit'89] Choquet-Bruhat, Y. and DeWitt-Morette, C.: Analysis, Manifolds and Applications, Part II, North-Holland (1989).

[Derd'92] Derdzinski, Andrzej: Geometry of the Standard Model of Elementary Particles, Text and Monographs in Physics, Springer (1992).

[Free/Uhle'84] Freed, D. S. and Uhlenbeck, K. K.: Instantons and Four-Manifolds, Mathematical Sciences Research Inst. Publ., Springer-Verlag (1984).

[Hig'64] Higgs, Peter: Phys. Rev. Lett. (12), 132, (1964).

[Koba/Nomi'96] Kobayashi, S. and Nomizu, K.: Foundations of Differential Geometry, Vol. I, Wiley Classics Library (1996).

[Nach'89] Nachtmann, O.: Elementary Particle Physics. Concepts and Phenomena, Springer (1989).

[Ster'95] Sternberg, Shlomo: Group Theory and Physics, Cambridge University Press, First paperback ed. (1995). 
[Tolk'03(a)] Tolksdorf J.: (Bosonic) mass meets (extrinsic) curvature, Journal Geom. Phys. (895), 1-21, (2003).

[Tolk'03(b)] Tolksdorf J.: (Fermionic) mass meets (intrinsic) curvature, to be published in Journal Geom. Phys.

[Trau'80] Trautman, A.: Fiber Bundles, Gauge Fields, and Gravitation, in General Relativity and Gravitation Vol. I, Edited by A. Held, Plenum Press, (1980).

[Wein'01] Weinberg, S.: The Quantum Theory of Fields, Vol. II, Cambridge University Press, (1996), Reprint 2001. 